\title{
AUSENGIA DE LIDERAZGO MOTIVACIONAL HACIA LA COMUNA EN LOS GOBIERNOS LOCALES EMERGENTES, PARA LA CONSTRUCCIÓN DE UNA VISIÓN DE DESARROLLO LOCAL Y HUMANO COMPARTIDA DE CARÁCTER SINÉRGICO E INCLUSIVO
}

\author{
LACK OF MOTIVATIONAL LEADERSHIP AT THE COMMUNE IN EMERGING LOCAL GOVERNMENTS \\ FOR THE CONSTRUCTION OF A SHARED LOCAL VISION AND SYNERGISTIC NATURE OF HUMAN \\ DEVELOPMENT AND INCLUSIVE
}

\author{
Juan Manuel Barreda Guerra* \\ barredaguerra@yahoo.es
}

\begin{abstract}
RESUMEN
La relevancia que están tomando los Gobiernos locales en el mundo globalizado de hoy en relación con el cambio de las necesidades de los vecinos, necesidades como salud, educación, trabajo, seguridad, protección del medio ambiente, territorialidad, inclusión de tecnología en los servicios administrativos, productivización de los recursos de la comuna mediante la productividad administrativa con transparencia, y actitud en el comportamiento organizacional e institucional alejada de los vicios como la corrupción, el favoritismo, y nepotismo y el sectarismo político interno en los municipios. Estos cambios de necesidades que se presentan en el día a día de las comunas de vecinos son cada día más fuertes y urgentes y ejercen mayor presión hacia los sistemas de gestión de los Gobiernos locales, manifestándose mayor exigencia en Gobiernos locales emergentes.

Los Gobiernos locales se ven hoy en la necesidad de generar estrategias de atracción de inversiones en sus territorios, desarrollar planes, programas y proyectos de inversión que pongan en valor sus recursos naturales y ventajas comparativas, a fin de que estas vayan generando sinergia económica social que impacte en la mejora y beneficio paulatino del desarrollo local y nivel de vida de su población.

Este tipo de necesidades han sido la base de inspiración de la ley de presupuesto participativo, para los efectos de dar cabida a los vecinos con sus propuestas de proyectos de desarrollo local basados en la atención de las necesidades de la población local, por ser ellos los que verdaderamente padecen y sufren la insatisfacción de sus necesidades sociales.
\end{abstract}

En muchas ocasiones, las autoridades políticas del Gobierno municipal han elaborado planes de gobierno en relación con la problemática actual del distrito. En la mayoría de planes de gobierno que se presentan en los Gobiernos locales se hace presente la visión y gestión edil de corto y mediano

\footnotetext{
Doctor en Ciencias Administrativas. Docente asociado de la Facultad de Ciencias Administrativas de la Universidad Nacional Mayor de San Marcos. Past Decano Nacional del Colegio de Licenciados en Administración del Perú. Ex Director Académico. Actualmente Director del Instituto de Investigación de la Facultad de Ciencias Administrativas.
} 
plazo. Esto se debe a que los tiempos de los Gobiernos municipales son verdaderamente cortos, en este sentido la visión de largo plazo solo cabe en la posibilidad de la reelección del alcalde. Sin embargo, uno de los requisitos más importantes para el desarrollo local es la continuidad de obras, desarrollo y formulación de nuevos proyectos que hayan sido propuestos con participación de los vecinos de la comuna y se hayan logrado incluir en el presupuesto concertado y a su vez este quede incluido en el presupuesto general del Gobierno local emergente, sobre todo tratando de aprovechar en estos proyectos las potencialidades con las que cuente la comuna, y que, en la medida de lo posible, "los proyectos existentes sean de carácter sinérgicos entre sí y ampliamente inclusivos, con el propósito de ir logrando el desarrollo local y el mejoramiento del nivel de vida de los vecinos de la comuna". Lamentablemente las propuestas de obras para el distrito emergente muchas veces está basada en las propuestas políticas del plan de gobierno del movimiento o partido político del alcalde electo. Muchas veces también estas obras son manejadas con acciones corruptas, con las que se pagan ciertos favores económicos para la ascensión al poder del alcalde. Cuando esto sucede, el factor de la prioridad de necesidades se desvanece y al mismo tiempo no se cuenta con mayores recursos para llevar adelante los proyectos que la comuna plantea.

Para el logro de estos propósitos, los líderes de las localidades deben ser preparados en espacios formales y de trabajo en sus territorios basados en el principio de "mandar obedeciendo", vale decir, respetando la cadena de mando de las órdenes, por estar estas basadas en los acuerdos decididos por la superioridad, en virtud de que estos acuerdos contienen a su vez los acuerdos de los vecinos lo cual significa que el poder, a más de orientarse al servicio de todos, sea también la manifestación del sentir de la voluntad de la comuna, tal como lo sostiene Giraldi Giulio1

Palabras claves: Liderazgo motivacional, presupuesto participativo, Gobierno local, Gobierno local emergente, desarrollo local y humano.

\section{ABSTRACT}

The relevance being taken by local governments in the globalized world of today in relation to the changing needs of the residents, needs ranging from health, education, work, security, environmental protection, territoriality, including technology services administrative, commoditization of resources of the district administrative productivity through transparent, and attitude in the organizational and institutional behavior away from vices such as corruption, favoritism and nepotism and internal political sectarianism in the municipalities. These changing needs that arise in day to day neighboring communes are becoming stronger and more urgent day and exert greater pressure on management systems of local governments, manifesting greater demand in emerging local governments.

Local governments are now in the need to develop strategies to attract investment in their territories, develop plans, programs and investment projects that give value to their natural resources and comparative advantages, so that these are generated social economic synergy that impact and gradual improvement in the benefit of local development and standard of living of its population.

Such needs were the basis of inspiration for the participatory budget law for the purposes of accommodating neighbors with their proposals for local development projects based on addressing the needs of the local population, be they the who truly suffer and suffer dissatisfaction social needs. Often the political authorities of the municipal government, government plans have been developed in relation to the current problems of the district; Most government plans presented in local governments is present mayor of vision and short and medium run, this time because of municipal governments are really short, in this sense the long view term only fit on the possibility of re-election of the mayor, However one of the most important requirements for local development is the continuation of works, development and formulation of new projects that have been proposed involving the residents of the community and have managed to include in the budget concluded while the longer stay included in the general budget of the emerging local government, especially trying to capitalize on these projects the potential for the instrument the municipality, and that the measures possible, "existing projects to be synergistic nature between them and, broadly inclusive, in order to be gaining Development Local and improving the standard of living of the residents of the municipalit Unfortunately the proposed works

1 GIRARDI, Giulio. Desarrollo Local Sostenible, Poder Local Alternativo y Refundación de la Esperanza. En: http://www.nodo50. org/venezuela-unida/art_giulio_girardi01.htm\#girardi01. Pág.15 
for the emerging district often is based on the policy proposals of the government plan of movement or political party elected mayor. Often these works are also handled with corrupt actions with certain economic favors to the rise to power of the mayor are paid. When this happens, the priority factor vanishes needs while there are no more resources to carry out the projects that the municipality raises. To achieve these goals the leaders of localities should be prepared in formal spaces and their work based on the principle of "obeying" territories, i.e. respecting the chain of command orders, being based on these agreements decided by the superiority under these agreements to contain in turn agreements neighbors which means that the prune, oriented over the service of all, is also the manifestation of the feeling of the will of the municipality, as Giulio Giraldi argues.

Keywords: Motivational leadership, Participatory budgeting, local government, emerging local government, Local and Human Development

\section{INTRODUCCIÓN}

En nuestro país existe significativa cantidad de distritos que han visto transcurrir años y décadas de su vida e historia a la espera de la construcción o tal vez renovación del puente que da acceso y salida al pueblo; de igual forma, años de espera de la población para la llegada de la energía eléctrica o el agua potable y la construcción del alcantarillado, décadas a la espera de la construcción de un pequeño policlínico, años de espera para que la escuela primaria del distrito o poblado pueda tener mayor cantidad de profesores, en virtud de que un solo profesor enseña ocho, nueve o tal vez todas las materias que se desarrollan en el grado que cursan los alumnos a su cargo, pudiendo ser estos sesenta, ochenta o más. Esta realidad se da en miles de escuelas en pueblos al interior del país; vale decir, la caótica y paupérrima educación primaria en nuestro país mediante la educación unidocente. De igual forma, en nuestro país tenemos centenas de distritos en los que podemos decir que generaciones de ciudadanos vecinos han vivido en sus distritos sin ver llegar agua, luz, servicios, pistas y veredas asfaltadas, parques de esparcimiento o áreas verdes para su población. En Lima tenemos distritos emergentes en los que sus pobladores, después de medio siglo de espera, recién pueden ver la avenida central del distrito asfaltada.

Lo descrito en el párrafo anterior no es más que la punta visible del iceberg. El problema es coyuntural, es parte del deficiente concepto y capacidad de gestión del Estado. Es también el producto de la desnaturalización del concepto de la vocación de servicio por el elemento político, mediante el cual se pone de manifiesto los intereses personales y partidarios o de grupo, antes que, el trabajo y usos de recursos de modo eficiente y eficaz, orientados en planes estratégicos de desarrollo para el mejoramiento de la calidad de vida de los ciudadanos de su comuna y el consecuente desarrollo local humano y la tan ansiada justicia social esperada por los pobladores.

Por lo expuesto, en nuestro país se hace necesaria la comunicación eficiente desde el Gobierno central, a efectos de motivar a los ciudadanos de todo el territorio nacional a participar en sus respectivos Gobiernos locales, mediante su presencia a las convocatorias al presupuesto participativo de su localidad.

\section{PLANTEAMIENTO DEL PROBLEMA}

Con relación al objetivo general de la investigación, que fue el medir si realmente es importante para quienes asumen la conducción de los Gobiernos locales emergentes promover y motivar la participación de los diversos actores sociales y económicos de la comuna en asuntos de suma importancia e interés para el desarrollo local y Humano del distrito, lo observado, explorado, medido y evaluado en la investigación nos ha hecho confirmar que efectivamente no es de sumo interés para quienes ejercen el Gobierno local, en virtud de que en primer orden esta la propuesta política de solución de los problemas del distrito, por la que han sido electos por los vecinos, y posterior a este hecho están las propuestas que pueden presentar los vecinos en el presupuesto participativo. Se abre también la necesidad de investigación con respecto a una sospecha razonable, en el sentido de que, tal vez, las nuevas autoridades electas no consideren conveniente ni beneficioso demasiada aproximación por parte de algunos actores sociales, políticos o económicos al plan de gobierno de los municipios en razón de algún tipo de celo.

En la fase exploratoria que se desarrollo en la investigación, se observó en cinco (05) distritos emergentes: Tres (03) de ellos de los distritos del Cono Norte y dos (02) del Cono Sur de Lima, 
ausencia de medios de comunicación que hubieran podido ser utilizados por estos municipios, como son: Boletines, folletos, revista municipales, medios electrónicos masivos páginas web, portales municipales, entre otros, con los cuales las municipalidades hubieran podido motivar y promover de mejor forma el interés de los vecinos de sus distritos para su participación vecinal como ciudadanos preocupados en la complejidad de la problemática de los asuntos ediles prioritarios del distrito en relación directa con el desarrollo local y humano de su distrito. Este hecho indicó a priori un pobre y escaso cumplimiento de la Ley General de Municipalidades, en la que se ordena promover la participación ciudadana del vecino en el Gobierno local, y a su vez nos permitió corroborar la existencia del problema planteado en la investigación.

En la observación realizada al interior de los locales municipales, se pudo advertir que, se exhibían tímidamente en sus vitrinas algunos folletos, diseñados al parecer en computadoras de las mismas municipalidades, en los que se instaba a los vecinos a tomar parte en el presupuesto participativo, para lo cual los vecinos que desearan concurrir debían estar inscritos en el padrón de participantes de las respectivas municipalidades, en caso contrario no podrían participar. Uno de los requisitos para poder participar era pertenecer a una determinada área del distrito y estar debidamente inscrito en alguna de las asociaciones, sólo de este modo podían inscribir algún proyecto, toda vez que otra institución de la zona o área no haya inscrito antes otro proyecto. En esa situación, el proyecto presentado sería solamente revisado, evaluado y comentado, dándose prioridad de tratamiento al proyecto que se presentó con fecha más antigua. Toda esta información fue hallada de manera diseminada y en hojas simples en vitrinas locales de los municipios, sin ningún poder de atracción a la vista de vecinos lectores.

Por estos hechos comunes en instalaciones municipales, se puede deducir que, siendo la comunicación un aspecto fundamental de carácter legal en lo concerniente a la aplicación y desarrollo de estrategias motivacionales dirigidas hacia la comuna a efectos de lograr de ella su más amplia y mayoritaria participación, la ausencia y deficiencia comunicacional pone en relieve la falta de liderazgo de las autoridades locales en el deseo de que participe la mayor cantidad de vecinos con ideas sobre determinados proyectos que puedan ser viables y coadyuven, impacten y beneficien el tan ansiado bienestar de los vecinos y comuna en general.

En la Municipalidad de Puente Piedra de modo circunstancial se pudo conversar con algunos vecinos, a quienes se les manifestó que nos encontrábamos realizando un estudio exploratorio referente a la participación de los vecinos en las convocatorias e invitaciones de su municipio a que participaran con la presentación de proyectos en los presupuestos participativos. Nos manifestaron que en alguna oportunidad habían participado con otros vecinos en una reunión de presentación de proyectos vecinales por la comuna, y que todos los proyectos que se presentaron en aquella oportunidad eran sobre pedidos de mejoras e instalaciones. Los proyectos solicitaban la construcción de veredas, iluminación de determinadas zonas del distrito, canchas de fulbito para los niños y jóvenes, instalaciones de red de agua y alcantarillado, fumigaciones, instalaciones de posta médica y puestos de vigilancia, entre otras necesidades comunales. Esta conversación permitió comprender que los vecinos están más preocupados por el mejoramiento de sus áreas o zonas del distrito en las que habitan. Verdaderamente este tipo de pedidos está muy lejos de que los vecinos conciban por sí mismos, la necesidad de generar proyectos de inversión según las potencialidades de su distrito. Las potencialidades y la construcción de oportunidades para poder aprovecharlas poniéndolas en valor requieren trabajarse capacitando a determinado sector de la población de vecinos, para que estos sean promotores de ideas sobre los proyectos de inversión, cuya formulación, evaluación y financiamiento para su gestión pueda ser viable y concordante con la visión compartida del desarrollo local y humano de la comuna. Ante estos hechos concretos, la investigación se planteó la interrogante sobre la existencia de:

\section{Problema}

Ausencia de liderazgo y motivación hacia la comuna por los Gobiernos locales emergentes para promover mayor participación vecinal en la construcción de una visión compartida de su desarrollo local y humano.

\section{OBJETIVO GENERAL}

Demostrar que los Gobiernos locales emergentes de Lima utilizan estrategias comunicacio- 
nales deficientes que, lejos de liderar y motivar a los ciudadanos a tomar parte en el presupuesto participativo, tienen a los diversos actores de la comuna en total estado de desinformación sobre cómo y quiénes pueden participar en él.

La investigación demuestra que el nivel de liderazgo y motivación aplicado por los Gobiernos locales emergentes de municipalidades de distritos de Lima Norte y Sur son totalmente deficientes, en relación con lo esperado por los vecinos y lo estipulado en la Ley General de Municipalidades, generando una condición que no permite la participación vecinal adecuada para la construcción de una visión compartida con participación de los diversos actores de la comuna.

\section{OBJETIVOS ESPECÍFICOS}

La investigación ha permitido demostrar que, las estrategias comunicacionales desplegadas por los gobiernos locales emergentes de los distritos de Lima Norte y Sur son escasas, y de muy poco alcance en su difusión hacia la comuna.

La investigación demuestra que los vecinos de los diversos distritos de Lima Norte y Sur se encuentran desinformados en cuanto a quiénes son los vecinos que pueden intervenir y cómo deben realizarse estas participaciones en las convocatorias al presupuesto participativo.

Las horas y fechas en las que se programan las sesiones en los locales municipales no facilitan la participación de los vecinos por razones laborales de estos, en alto porcentaje.

\section{JUSTIFICACIÓN}

La investigación ha contribuido a confirmar que los Gobiernos locales emergentes no lideran ni motivan adecuadamente la participación de sus vecinos con sus iniciativas, para que estos presenten proyectos de inversión que pudieran ser considerados en el presupuesto participativo que deba integrarse al presupuesto integral del distrito, a efectos de que estos proyectos, en su desarrollo y gestión, contribuyan en mejorar el desarrollo local y humano de la comuna.

La investigación se encuentra justificada al haber permitido demostrar que los vecinos de los Gobiernos locales emergentes no cuentan, en muy alto porcentaje, con la información necesaria en cuanto a fechas y horas programadas y requisitos para participar en las sesiones de tratamiento del
Presupuesto Participativo distrital.

Servirá de base técnica para sugerir a los Gobiernos locales emergentes que elaboren planes educativos y de capacitación a su comuna sobre la importancia del aspecto de la asociatividad vecinal y representación ciudadana para participar en el presupuesto.

Permitirá elaborar una propuesta al Ministerio de Educación para que el canal del Estado comunique de manera reiterativa, a los vecinos de los diversos distritos emergentes del país, las fechas, horas y lugares en los que se programarán las sesiones para el presupuesto participativo del respectivo distrito.

Nos enseña que debemos educarnos en el concepto de que el desarrollo es un tema de participación general y no únicamente político. Debemos autoinculcarnos la cultura de la representatividad y la participación ciudadana como aporte a la solución de los problemas comunes.

\section{HIPÓTESIS}

\section{Hipótesis general}

Los pobladores de distritos de Lima Norte y Sur no intervienen en el presupuesto participativo de su municipalidad por la ausencia de liderazgo y motivación de las autoridades municipales hacia sus comunes.

\section{Hipótesis específicas.}

Los ciudadanos de los distritos de Lima Norte y Sur no participan en el presupuesto participativo por desconocimiento y porque el horario de sus obligaciones y responsabilidades laborales no les permite.

Los vecinos no participan en el presupuesto participativo por mala comunicación, desinformación y percepciones negativas sobre la autoridad municipal.

\section{METAS ESPECÍFICAS}

Verificar si realmente a los consejos de los Gobiernos locales emergentes en turno les preocupa e interesa la amplia y real participación ciudadana en asuntos de sumo interés de la comuna para el mejoramiento del gobierno local y humano en el distrito.

Demostrar que el uso de estrategias de comunicación deficientes en la convocatoria de la comuna a su participación en el presupuesto 
participativo es un indicador de desinterés de los gobiernos locales de turno en hacer participar a la comunidad de manera democrática en la construcción de una visión compartida.

Plantear una propuesta al Gobierno nacional para la supervisión de la eficiencia de la estrategia comunicacional en las convocatorias a la participación vecinal por los Gobiernos locales emergentes.

\section{MARCO TEÓRICO DEL TRABAJO}

El marco teórico para la presente investigación parte de una revisión bibliográfica para definir un marco teórico general sobre el liderazgo político en la gestión municipal. Tomando como referencia el marco teórico y las preguntas de investigación, se da inicio a la exploración bibliográfica. En ella se pudo encontrar que la Unión Europea cuenta con el Fondo Europeo de Desarrollo de la Sociedad Civil, que en trabajos conjuntos con países latinoamericanos auspicia programas de apoyo a las iniciativas locales de la sociedad civil, para de este modo cooperar, ayudar y orientar a las organizaciones de la sociedad civil, como en el caso de República Dominicana, en donde interviene apoyando el propio desarrollo institucional en el aspecto individual como de redes organizadas que estas sostienen con las autoridades de la administración pública de los contextos locales y central para el desarrollo económico y social de sus comunidades ${ }^{1}$.

El objetivo de estos estudios es fortalecer las organizaciones de la sociedad civil en la dinámica de sus planes de desarrollo y presupuesto de sus comunas, abarcando para ello cuatro areas de acción de modo estratégico:

1. Fortalecimiento de las capacidades locales.

2. Fortalecimiento de las organizaciones locales.

3. Control ciudadano e integración de actores claves vulnerados en sus derechos.

4. Observancia de la democracia local. Como forma de observación de procesos de participación ciudadana, democracia y desarrollo local.

Max Weber plantea tres tipos de dominación:

1. Legal.- En función de la presencia del Estado.
2. Tradicional.- La sociedad acepta la existencia de un orden social en función de reglas.

3. Carismática.- Fuerza de seducción y sometimiento que puede poseer una persona.

La primera y tercera son las comunes en la realidad latinoamericana. Pero hoy en día la experiencia nos va mostrando otro tipo de liderazgo que demanda la sociedad: el de la credibilidad y los resultados.

Países de regiones y zonas más desarrolladas del planeta observan que en Latinoamérica existen ciudades, Gobiernos locales, regionales y países que permanecen estáticos en su desarrollo local y humano en decenas de años, sin ningún crecimiento ni desarrollo, aun cuando estas han tenido igualmente decenas de periodos de gobiernos, y en ellas han transcurrido generaciones, sin que estas hayan podido alcanzar algún grado mejoramiento en su desarrollo local y humano. Debido a este factor, explican la orientación de su apoyo hacia America latina y buscan inculcar la toma de conciencia en la comuna de la necesidad de avanzar en función de la participación ciudadana, mediante la búsqueda de resultados económicos y sociales concretos ${ }^{2}$.

La presencia de estos esfuerzos de organizaciones transnacionales bajo modalidad de ONGs se da en Perú en muchísimas regiones, advirtiendo esto que en nuestro país existe gran ausencia de de liderazgo y motivación desde los gobiernos locales a fin de motivar la participación y fortalecimiento de las organizaciones sociales en el gobierno y progreso de las comunas de los gobiernos locales emergentes.

\section{El buen gobierno local facilita el desarrollo humano y sustentable}

"En cada país las normas y costumbres determinan las características y funciones de sus Gobiernos locales. Sin perjuicio de la manera en que estén organizados y su dotación efectiva de recursos, concebimos a los Gobiernos locales como una instancia autónoma del Estado cuya función principal es la promoción y facilitación de un desarrollo local humano y sustentable" (Agenda Municipal Latinoamericana) ${ }^{3}$.

\footnotetext{
1 Fundación Solidaridad. Brochure Informativo. Proyecto Participación e Incidencia de la Sociedad Civil en la Gestión Municipal

2 Rosa Arlene María. Estudio Sobre Buenas Practicas de Liderazgo Político en la Gestión Municipal en República Dominicana. República Dominicana 2009.

3 Agenda Municipal Latinoamericana. Evento desarrollado del 28 a 29 de noviembre del 2011 en la ciudad de San Francisco de Milagro- Ecuador
} 
La base de la identidad es la cultura. Esta es la única garantía de continuar siendo nosotros en un mundo económico que se expande bajo el modelo de la globalización. Los esfuerzos que se realicen en función de rescatar nuestra identidad son hoy responsabilidades mayores de los Gobiernos locales en virtud de que este rescate brinda enormes ventajas en el desarrollo económico local.

Tarea municipal importante es, entonces, la preservación de la cultura. En un mundo globalizado que arrincona las identidades locales, es menester preservar sistemáticamente nuestro patrimonio cultural. ¿Qué puede hacer la municipalidad? He aquí algunos ejemplos que Mario Rosales $^{4}$ cita en su libro El Buen Gobierno Local y qué hacer para liderarlo:

La Prefeitura de Curitiba, en Brasil, se ha hecho famosa por un ambicioso programa de ordenamiento y valorización de su ciudad, que contempla un efectivo plan de transporte, el rescate de la arquitectura urbana y su presentación valorizada a los ojos del visitante.

En Quito, Ecuador, declarada por UNESCO "Patrimonio Cultural de la Humanidad", las edificaciones antiguas del centro son mantenidas e iluminadas y su arquitectura no puede ser alterada, por ordenanza municipal. Muchos municipios -como Sucre en Bolivia o Cartagena de Indias en Colombia- desarrollan políticas similares de protección del patrimonio urbanístico local y dictan ordenanzas para regular su uso. Valparaíso, Chile, es una de las últimas ciudades declaradas patrimonio histórico por la UNESCO.

En Chile, la pequeña Municipalidad de Hijuelas organizó un concurso de leyendas locales, mediante el cual los jóvenes rescataron mitos y leyendas de la tradición oral de los ancianos; además de publicarlos, los mejores cuentos recibieron premios especiales. De modo similar, la Municipalidad de La Ligua organizó un museo arqueológico, sobre la base de miles de piezas rescatadas y clasificadas por los jóvenes del Liceo de la localidad; todo un esfuerzo dirigido y animado por un profesor municipal.

En ciudades y pueblos de la sierra peruana y ecuatoriana, tradicionalmente se realiza la fiesta anual de la localidad con carros alegóricos, concursos de baile, trajes típicos, exposiciones y premios. A menudo se incluye un concurso de comida típica, con jurado seleccionado entre los mejores catadores y comedores de la localidad.

Las comunas de Santiago de Chile, todos los veranos, realizan conciertos de música al aire libre y teatro, lo que ha pasado a ser una tradición que dura ya décadas y que reúne y educa sobre todo a la población joven.

En San Carlos, Uruguay, la junta local -derivación descentralizada de la intendencia municipal- mantiene y alimenta la tradición oral acerca de las características positivas del habitante de la localidad: "el "carolino" es emprendedor y siempre vuelve a su tierra" afirman siempre sus autoridades a quienes los visitan. Otro tanto ocurre con el concepto de emprendedores que tienen los habitantes de Rafaela, Argentina, o de La Ligua, en Chile.

En muchos pequeños pueblos de América Latina -entre ellos los famosos Otavalo en Ecuador y Chichicastenango en Guatemala- hay mercados de artesanía local artística. En algunas ciudades latinoamericanas han aparecido "pueblitos típicos" o "casonas" para cumplir la misma función.

En las ciudades grandes e intermedias de América Latina se están estableciendo cada vez más áreas peatonales, con incentivo especial actividades culturales como teatro callejero y la presentación de artistas locales.

Como podemos apreciar, son muchos Gobiernos locales en Latinoamérica que van a la vanguardia en el rescate de su identidad cultural, a fin de poder protegerla y sostenerla. Esta nueva conducta que deben asumir los Gobiernos locales con potenciales históricos que pueden ser puestos en valor tiene luego un interesante impacto en el entorno económico de las localidades. Así mismo podemos advertir la preocupación de las autoridades de estos gobiernos locales y su liderazgo sobre sus poblaciones para motivarlas y estimularlas en la presentación de iniciativas, y en recogerlas de la población y traducirlas en proyectos para su evaluación y posteriormente gestionar proyectos concretos.

\section{Nuevas formas de gestión local}

La búsqueda continua de buenas prácticas de desarrollo local nos comprueban que los esfuerzos por la descentralización han abierto nuevas áreas

4 Mario Rosales en su libro El Buen Gobierno Local y qué hacer para liderarlo. Pag 51 
y espacios de autonomía y gestión dinámica para el desarrollo local, lo que estimula la innovación de la gestión y permite la presencia de nuevas y mejores formas de hacer y lograr los resultados propuestos.

Si bien las reformas descentralizadoras asignan importancia a los niveles locales de gobierno, los mayores recursos otorgados son exiguos. Los Gobierno locales presentan un incremento promedio en América Latina del $12 \%$ al $18 \%$, mientras que el nivel promedio del gasto del gobierno central se mantiene en un promedio del orden del $82 \%$.

Pero las comunidades locales asumen efectivamente el mensaje descentralizador e incrementan sus demandas por mayor democracia, participación, inversión y mejor extensión y calidad de los servicios. Los diversos objetivos de la descentralización -incluso siendo contradictorios entre sí- se traducen en un aumento acumulativo de las demandas hacia las autoridades locales.

Muchas autoridades territoriales asumen el poder otorgado por los electores de manera más creativa e innovadora. Como afirmara Tito Choca$\mathrm{no}^{5}$, ex alcalde de Tacna, "los límites del quehacer del alcalde (están establecidos) por su creatividad". No se trata de forzar o no respetar la ley, es necesario buscar nuevos espacios y maneras de incrementar los medios municipales para ampliar su radio de influencia y mejorar su performance ${ }^{6}$.

\section{Asociatividad bajo liderazgo y motivación a la comuna}

Alonso Gamero ${ }^{7}$, del Instituto Universitario de Tecnología del estado de Falcón, en su informe presentado al proyecto (Programa Nacional de Formación en Administración), de acuerdo a las líneas maestras del plan de desarrollo económico y social de la nación 2007 - 2013, manifiesta como una de sus conclusiones que la realidad en las comunidades está relacionada con el mismo, especialmente con las directrices contenidas en el plan, en donde se señala que la "suprema felicidad social" tiene como objetivo la inclusión social. Esto es fácilmente advertido en las iniciativas de formación de los consejos comunales, las misiones y la posibilidad de ingresar a los programas de alimentación u otros tipos de posibles recursos que satisfagan las necesidades de los vecinos de una comuna. Es importante destacar el liderazgo que ejercen las autoridades ediles y los dirigentes comunales en el esfuerzo de aplicar la "democracia protagónica revolucionaria”, que tiene como propósito lograr que los individuos se sensibilicen y organicen alrededor de una actitud de logro y ventajas que permitan la unión y cohesión social mediante la asociatividad de modo cooperativo, vale decir, transformar las debilidades personales en fortalezas comunales asociativas.

El proyecto tiene por nombre Plan de Organización para el Funcionamiento Óptimo del "Consejo Comunal Curazaíto I" del Municipio Miranda del Estado Falcón. El proyecto viene siendo trabajado con la finalidad de colaborar con el consejo comunal para un funcionamiento exitoso. La vinculación del proyecto con el área de conocimiento en el subsistema del proceso administrativo se considera fundamental para una organización en la que sus participantes se unan y formen para lograr obtener los cuatro componentes básicos como son: el liderazgo, la motivación, la comunicación y la integración, para poder llevar a cabo en forma eficaz y eficiente sus proyectos y, con ello, el logro de la tan ansiada inclusión social.

Como podemos advertir en trabajos realizados sobre estas variables en otras latitudes del mundo, el liderazgo y la motivación al igual que el factor de comunicación son fundamentales en la búsqueda de resultados positivos en la organización de la comuna y su orientación hacia proyectos y programas con los que la población pueda lograr solucionar problemas comunes en ella y en la asociatividad encuentre una modalidad de fortalecerse socialmente para hacer frente a sus debilidades individuales, y lograr, de algún modo, un mejor camino hacia un futuro con mayores posibilidades para su desarrollo humano.

\section{MÉTODO}

Se inició la investigación con el uso de metodologías exploratorias y desde luego descriptivas-causales a efecto de medir frecuencia y su relación con los hechos. Las fuentes principales de información como son los municipios han sido el área de investigación que ofreció mayor cantidad de dificultades para poder acceder a

\footnotetext{
5 Citado en "Los Secretos del Buen Alcalde", M. Rosales, IULA., Quito, 1993. Página 39.

6 Jaime Ravinet, ex alcalde de Santiago, afirmaba que el rol de los abogados municipales no es decir a las autoridades que "no se puede", sino indicar la mejor manera de llevar adelante las decisiones políticas de acuerdo al marco legal.

7 “Alonso Gamero" (IUTAG), 2011Santa Ana de Coro: Instituto Universitario de Tecnología “
} 
la data necesaria. No todas las municipalidades inician su trabajo de diseño y programación de comunicación en las mismas fechas, por tanto las acciones que planifican para llevar a efectos sus comunicaciones difieren unas de otras. De igual forma, el personal que puede dar información, por lo general, es personal contratado, cuyo horario de ingreso difiere del horario institucional y su labor es más de campo, razón por la que se hace difícil contactar personalmente. Se puede decir que casi no se encuentran en el municipio, dado que se debe entender que su trabajo comunicacional es externo.

La investigación plantea hipótesis explicativas de tipo estocásticas, y efectos predictivos.

Para los efectos de obtener valores que permitieran comprobar las hipótesis se utilizó métodos cuantitativos muestrales, con uso de pilotos previos. Para la selección del área territorial del muestreo, se utilizó el método aleatorio.

Se seleccionó de modo aleatorio un distrito de la zona Lima norte. En la selección aleatoria (sorteo) salió el distrito de Comas.

POBLACIÓN: Hombres y mujeres mayores de 18 años del distrito de Comas.

MUESTRA: Siendo la población estudiada de dimensión infinita, se ha utilizado la fórmula usada por la universidad de Harvard para esos tamaños de población.

La muestra fue determinada dentro del nivel de confiabilidad $\mathrm{N}_{\mathrm{c}}=95 \%$

El valor presumible de la ocurrencia del fenómeno se estimó en $\mathrm{P}=5 \%$

El valor presumible de la no ocurrencia del fenómeno se determinó por $(1-\mathrm{P})=95 \%$

El erro estimado para la determinación del tamaño de la muestra se determinó inicialmente en $5 \%$. A fin de contar con un margen de error inferior al trabajado, se amplió el tamaño.

La desviación estándar correspondiente al $\mathrm{N}_{\mathrm{c}}$ es igual a la varianza al cuadrado $(2)^{2}=4$

Con utilización de la fórmula $\mathrm{n}=\sigma^{2}[(\mathrm{P})(\mathrm{Q})]$ / $\mathrm{e}^{2}$ se determinó un tamaño de muestra

$\mathrm{n}=76$
Con el propósito de disminuir el margen de error a (4.35\%), se amplió el tamaño de la muestra a 100 entrevistas.

$\mathrm{n}=100$ encuestas.

Se selecciono el distrito a encuestar de forma aleatoria (Comas).

Para la aplicación de la muestra a estudiar, se diseñó un cuestionario cuyo objetivo estuvo basado en obtener información sobre las variables en estudio, como: comunicación, conocimientos sobre requisitos para la participación en el presupuesto participativo, disponibilidad de tiempo, asociatividad, ocupación, confiabilidad en la autoridad edil.

Las condiciones base para ser considerado unidad estadística de información válida fueron:

- Varón o dama mayor de 18 años.

- Ser vecino del distrito.

- Que las unidades estadísticas de información UEI procedieran de áreas del distrito que guarden suficiente dispersión entre ellas.

Se capacitaron cinco personas en el manejo y aplicación del cuestionario para el momento de la entrevista.

Se capacitó una persona para la ejecución y la supervisión del trabajo de campo.

Se capacitaron dos personas para la tabulación, cálculos y elaboración de cuadros sobre los datos obtenidos.

Se diseñó el cuestionario a utilizar en el trabajo de campo de la investigación en función al objetivo de captar informaciones referentes a las variables contenidas en el problema y objetivos de la investigación, así como también el nivel de conocimiento por parte de la población encuestada sobre quiénes son los vecinos que pueden participar en el presupuesto participativo, la eficiencia de los métodos de comunicación utilizados por las municipalidades, disponibilidad de tiempo del vecino para poder participar en las convocatorias, el uso del liderazgo y la motivación en las comunicaciones de la municipalidad. 
RESULTADOS

\section{Resultados observados en el muestreo}

CUADRO No 1

\begin{tabular}{|l|c|}
\hline \multicolumn{2}{|l|}{$\begin{array}{l}\text { Pregunta: ¿Cómo se enteró usted sobre el presupuesto partici- } \\
\text { pativo de su municipalidad?. }\end{array}$} \\
\hline Por comentarios de otras personas & $85 \%$ \\
\hline $\begin{array}{l}\text { Lo lei en banderolas, gigantografías, volantes, trípti- } \\
\text { cos, folletos, afiches que dan en la municipalidad }\end{array}$ & $15 \%$ \\
\hline T O T A L : (BASE 100 Encuestas) & $100 \%$ \\
\hline
\end{tabular}

Fuente: Elaboración propia.

Como puede apreciarse en los resultados que presentó la encuesta, más se entera la gente en conversaciones particulares. Este porcentaje es de $85 \%$, siendo esta cifra casi el séxtuplo en porcentaje sobre los del efecto comunicacional que tuvieron los medios utilizados por la municipalidad, los cuales alcanzaron el 15\%. Las cifras evidencian la ineficiencia del método comunicacional usado por la municipalidad.

\section{HIPÓTESIS}

$\mathrm{H}_{\mathrm{o}}$ La estrategia de comunicación efectuada por las municipalidades es altamente efectiva

$\mathrm{H}_{1}$ La estrategia de comunicación efectuada por las municipalidades es deficiente

Nivel de significancia 5\%

Uso de distribución $\mathrm{X}^{2}$

Grados de libertad $\mathrm{K}=2-1$ dado $\mathrm{m}=0$

Decisión: Rechazar $\mathrm{H}_{\mathrm{o}}$ si X $\mathrm{X}^{2}>3.841$

$X^{2}=289>3.841$. Se acepta $H_{1}$

\section{CUADRO No 2}

\begin{tabular}{|l|c|}
\hline \multicolumn{2}{|l|}{$\begin{array}{l}\text { Pregunta: ¿Sabe quiénes pueden participar en los presupues- } \\
\text { tos participativos de la municipalidad de su distrito? }\end{array}$} \\
\hline No sabe & $78 \%$ \\
\hline Sí sabe & $22 \%$ \\
\hline T O T AL : (BASE 100 Encuestas) & $100 \%$ \\
\hline
\end{tabular}

Fuente: Elaboración propia.

Una cifra muy alta que alcanza el $78 \%$ de la población desconoce quiénes pueden participar, y tan solo alrededor de un quinto de la muestra sabe quiénes pueden participar.

Ho Los vecinos saben muy bien quién puede participar en el presupuesto participativo.
H1 Los vecinos no saben quiénes pueden participar en el presupuesto participativo.

Nivel de significancia 5\%

Uso de distribución X2

Grados de libertad $K=2-1$ dado $m=0$

Decisión: Rechazar Ho si X2 > 3.841

X2 $=1568>3.841$. Se acepta H1

\section{CUADRO No 3}

\begin{tabular}{|c|c|}
\hline \multicolumn{2}{|c|}{$\begin{array}{l}\text { ¿Qué necesita principalmente para poder participar en el } \\
\text { presupuesto participativo de la municipalidad? }\end{array}$} \\
\hline Más y mejor información sobre el tema & $71 \%$ \\
\hline Disponer de tiempo & $29 \%$ \\
\hline T O TA L: (BASE 100 Encuestas) & $100 \%$ \\
\hline
\end{tabular}

Fuente: Elaboración propia.

El cuadro muestra que los vecinos del distrito de Comas requieren de mayor y mejor información, $71 \%$ de los entrevistados dieron esta opinión. Mientras, el $29 \%$ atribuyó razones de tipo personal, ya que requerían de mayor disponibilidad de tiempo para poder asistir a los talleres de entrenamiento previo que ofrece la municipalidad a la participación en las reuniones de trabajo para el presupuesto participativo.

$\mathrm{H}_{\mathrm{o}}$ Los vecinos no necesitan información ni tiempo para participar en el presupuesto participativo

$\mathrm{H}_{1}$ Los vecinos requieren de mayor información y disponibilidad de tiempo para participar en el presupuesto participativo.

Nivel de significancia $5 \%$

Uso de distribución $\mathrm{X}^{2}$

Grados de libertad $K=2-1$ dado $m=0$

Decisión: Rechazar $\mathrm{H}_{\mathrm{o}}$ si $\mathrm{X}^{2}>3.841$

$\mathrm{X}^{2}=882>3.841$ Se acepta $\mathrm{H}_{1}$

\section{CUADRO No 4}

\begin{tabular}{|c|c|}
\hline \multicolumn{2}{|c|}{$\begin{array}{l}\text { Pregunta: ¿Pertenece usted a alguna organización social } \\
\text { (junta vecinal, asentamiento humano, asociación de vecinos, } \\
\text { organización de mujeres, de productores, gremio laboral, orga- } \\
\text { nización de jóvenes, etc.) en su distrito? }\end{array}$} \\
\hline No pertenezco a ninguna organización & $81 \%$ \\
\hline Sí pertenezco a una organización & $19 \%$ \\
\hline TO TAL: (BASE 100 Encuestas) & $100 \%$ \\
\hline
\end{tabular}

Fuente: Elaboración propia. 
El distrito de Comas es un distrito con baja organización social, tan solo el $19 \%$ pertenece a una organización social. Este bajo nivel de formalidad en su población disminuye la fuerza de las propuestas o de los proyectos. El $81 \%$ de la población se encuentra desarticulada, vale decir, la población carece de formalidad de representación.

$\mathrm{H}_{\mathrm{o}}$ Los vecinos de la comuna están organizados en asociaciones.

$\mathrm{H}_{1}$ Los vecinos de la comuna no están organizados en asociaciones.

Nivel de significancia 5\%

Uso de distribución $\mathrm{X}^{2}$

Grados de libertad $\mathrm{K}=2-1$ dado $\mathrm{m}=0$

Decisión: Rechazar $\mathrm{H}_{\mathrm{o}}$ si $\mathrm{X}^{2}>3.841$

$\mathrm{X}^{2}=3042>3.841$ se acepta $\mathrm{H}_{1}$

\section{CUADRO No 5}

\begin{tabular}{|l|c|}
\hline \multicolumn{2}{|l|}{ Dígame, por favor. ¿en qué horario realiza su trabajo? } \\
\hline Horario de 8.00 AM a 5. 30 PM & $90 \%$ \\
\hline Horario Variado & $10 \%$ \\
\hline T O TA L. (BASE 100 Encuestas) & $100 \%$ \\
\hline
\end{tabular}

Fuente: Elaboración propia.

El cuadro demuestra que, siendo las reuniones de trabajo para el presupuesto participativo durante el horario en el que la gran mayoría de los vecinos se encuentran trabajando, esto es un impedimento para que puedan asistir. $90 \%$ trabaja en horario común de trabajo, de 8 AM a 5 PM. $\mathrm{Si}$ a los porcentaje obtenidos se les cruza con los porcentajes sobre los perfiles ocupacionales de las personas que manifestaron que requieren disponer de tiempo, podemos observar que, de las 29 personas que tienen tiempo para poder participar en el Presupuesto Participativo (Ver cuadro $\mathrm{N}^{\circ} 3$ ), (10) serían trabajadores independientes, siendo estos los que tal vez puedan disponer de tiempo, y las personas restantes son (5) obreros, (5) empleados, (7) amas de casa y (2) con otros tipos de empleos, quienes tal vez podrían tener tiempo después de su horario de trabajo.

$\mathrm{H}_{\mathrm{o}}$ Los vecinos de la comuna pueden participar en las reuniones para el presupuesto participativo en cualquier momento.

$\mathrm{H}_{1}$ Los vecinos de la comuna no pueden participar en el presupuesto participativo en las fechas y horarios que se programa.

Nivel de significancia $5 \%$

Uso de distribución $\mathrm{X}^{2}$

Grados de libertad $K=2-1$ dado $m=0$

Decisión: Rechazar $\mathrm{H}_{\mathrm{o}}$ si $\mathrm{X}^{2}>3.841$

$X^{2}=3200>3.841$ Se acepta $H_{1}$

CUADRO $\mathrm{N}^{\circ} 6$

\begin{tabular}{|l|c|}
\hline \multicolumn{2}{|l|}{ Pregunta: ¿Cuál es su ocupación? } \\
\hline Independiente & $34 \%$ \\
\hline Obrero & $19 \%$ \\
\hline Empleado & $19 \%$ \\
\hline Su casa & $24 \%$ \\
\hline Otros & $4 \%$ \\
\hline T O TAL: (Base 29 personas) Cuadro $\mathrm{N}^{0} 3$ & $100 \%$ \\
\hline
\end{tabular}

Fuente: Elaboración propia.

El cuadro $\mathrm{N}^{\circ} 6$ muestra la cantidad de personas en porcentajes que desearían participar en el presupuesto participativo, podemos observar que el $34 \%$ son personas con trabajos independientes, en las que cabría la posibilidad de poder adecuar u organizar sus tiempos disponibles para poder asistir a las sesiones que convoca la municipalidad. Un significativo porcentaje de 62 $\%$ tendría ciertas dificultades para poder asistir en horarios normales de trabajo, dado que estas personas son amas de casa, empleados y obreros, cuya responsabilidad en su horario de trabajo no se lo permitiría.

CUADRO No 7

\begin{tabular}{|l|c|}
\hline $\begin{array}{l}\text { ¿Perfil de quiénes y cuántos tienen posibilidades } \\
\text { de tiempo para poder participar en los } \\
\text { presupuesto participativos? }\end{array}$ & Porcentaje \\
\hline Independientes & 10 \\
\hline Obreros & 5 \\
\hline Empleados & 5 \\
\hline Su casa & 7 \\
\hline Otros & 2 \\
\hline T O TA L: (BASE 29 Encuestas). & \\
\hline
\end{tabular}

Fuente: Elaboración propia. 
En el cuadro que antecede se puede observar la cantidad de personas y sus ocupaciones que fueron halladas en la muestra utilizada. Diez personas con trabajo independiente, diecinueve personas con trabajo sujetos a dedicación por la labor que efectúan. Dos personas manifestaron tener horarios muy variados. Lo que se puede colegir de esta realidad es que esta cantidad de personas no tendrían representatividad de la problemática.

\section{CUADRO No 8}

\begin{tabular}{|l|c|}
\hline \multicolumn{2}{|l|}{ ¿Tiene confianza en el actual alcalde del distrito? } \\
\hline No tienen confianza. & $89 \%$ \\
\hline Tienen algo de confianza & $3 \%$ \\
\hline Sí tiene confianza & $8 \%$ \\
\hline T O TAL :(BASE 100 Encuestas) & $100 \%$ \\
\hline
\end{tabular}

Fuente: Elaboración propia.

Es sorprendente el alto porcentaje en una comuna que no confía en su autoridad edil, el 89\% tiene reparos en brindar su confianza. Tal vez el hecho se deba a lo poco que han podido efectuar de sus promesas electorales los alcaldes elegidos en los distritos emergentes. Tan sólo un $11 \%$ confía algo en sus autoridades ediles, este aspecto lo dice todo para la investigación: la gente no confía en su autoridad edil. En este sentido, es lógico entender que los alcaldes no ejercen liderazgo en sus poblaciones y mucho menos pueden motivar a sus comunas a que estas sean más participativas en la construcción de una visión participativa del desarrollo humano y local. Y mucho menos a generar ideas comunales para proyectos de inversión con carácter sinérgico e inclusivo.

Ho Las autoridades ediles gozan de plena confianza por parte de los vecinos

H1 Las autoridades ediles no gozan de la confianza por parte de los vecinos

Nivel de significancia 5\%

Uso de distribución X2

Grados de libertad $\mathrm{K}=3-1$ dado $\mathrm{m}=0$

Decisión: Rechazar Ho si X2 > 5.991

X2 $=4660.67>5.991$ Se acepta H1.

\section{DISCUSIÓN}

El presupuesto participativo es un mecanismo de participación democrática de la comuna, en el cual esta puede formular propuestas y planes para conseguir mejoras en el entorno del desarrollo local y humano. La promoción y motivación a los vecinos para su concurrencia a las convocatorias de participación deben ser fuertemente publicitadas por las municipalidades, sin embargo, a las autoridades ediles en turno parece no importarles mucho la participación vecinal en los asuntos y problemas de la comuna.

La comuna tiene el derecho inalienable de acceso a la información y transparencia en el uso de los recursos de las autoridades ediles y a su presencia ante la rendición de cuentas a los vecinos sobre la acción pública en ejercicio. De igual forma, debe hacer público el estado económico financiero de la municipalidad y compromisos asumidos tanto por las autoridades y la sociedad civil del distrito sobre el destino de la comuna, sin embargo este hecho no se da en los Gobiernos locales emergentes.

Parecería que las estrategias de comunicación que utilizan los Gobiernos locales emergentes son distantes a fines convocatorios masivos. Los ciudadanos de la comuna, aparte de estar desinformados sobre fechas, lugares, horas apropiadas para poder concurrir y temas a tratar en los presupuestos participativos que se realizarán, en muy corto plazo pierden la confianza en sus autoridades ediles en función de situaciones que se dan en el manejo económico de la gestión edil en turno. La urgencia y prioridad de las necesidades insatisfechas de la comuna parece no ser la fuente de inspiración de los planes de desarrollo local y de mejoramiento de la calidad de vida de los ciudadanos.

Es urgente motivar a la población desde otros medios comunicacionales que tenga el Estado, a efectos de interesar a los vecinos en la participación ciudadana en el presupuesto participativo e informar permanente sobre la marcha y los logros de la gestión interna de los municipios locales emergentes, y cómo estos impactarán en la mejora de la calidad de vida de la comuna.

El presupuesto participativo no es la solución ideal de todos los problemas de los Gobiernos locales emergentes ni de los residenciales, empero, sí es un medio altamente positivo para 
la democracia y el poder local, en el cual se pueden desarrollar estrategias de cogobierno y "cogestión", para que los representantes ediles y las comunas participen de manera voluntaria y tomen decisiones cada vez más próximas con los intereses de la mayoría de las comunas en su búsqueda permanente del tan ansiado desarrollo local sostenido y mejoramiento de la calidad de vida de sus ciudadanos.

\section{CONCLUSIONES}

Perú es un país con altísima desorganización social. En contextos más amplios como los vecinales, hoy en día vivimos sin conocernos ni saludarnos con los vecinos con los que vivimos en la misma cuadra, a tan solo tres o cuatro casas más allá de la nuestra. Nos estamos enfriando socialmente, estamos caminando hacia un modelo de convivencia y comportamiento social de tipo americano o europeo. Estamos copiando mal el ejemplo, ellos lo hicieron después de haber logrado el desarrollo económico, social y tecnológico de sus sociedades y países. Nosotros estamos saltando esas etapas, no contamos con ninguno de los tres desarrollos alcanzados por ellos.

El nivel de desorganización social alto es peligroso en toda sociedad, esto da lugar a ventajas al elemento político, ya que en la sociedad desorganizada en la que interactúa puede engañar fácilmente con gran habilidad y destreza mediante falsas promesas, y una vez que el elemento político logra su acceso al poder, simplemente se olvida de lo ofrecido y procurará de algún modo el alejamiento de sus electores, sea esto a nivel local, regional o nacional. El político pretende aplicar como plan o programa de gobierno lo que del análisis y conclusión sobre la realidad encontrada en el cargo al que postuló, le permita hacer cosas en las que sus intereses, que van desde lo personal hasta lo partidario, le permitan beneficios personales de cualquier orden. Lo que se le ofreció al pueblo en la campaña no es posible realizarlo; y si lo es, pues se hará muy poco o algo. Los recursos hay que destinarlos hacia algo más rentable y provechoso para los grupos de interés de su entorno, pudiendo ser los del partido o agrupaciones políticas, económicas o sociales con las que accedió al poder.

Tras el olvido de lo ofrecido por los políticos, a estos no les es tan conveniente tener al pueblo participando en el gobierno. Hay que enfriar al elector y dejarlo caer en la desilusión, hasta el punto en que este diga que se equivocó y termine diciendo yo voté por tal o cual, y este o estos no hacen nada de lo que ofrecieron para solucionar los problemas en los que nos encontramos. Este análisis lo hace el ciudadano de modo individual y termina en la desilusión y espera de otro proceso electoral, en el cual vuelva a equivocarse.

Una vez electos, los alcaldes y autoridades ediles no ejercen liderazgo en sus comunas para la participación de los ciudadanos, empresarios e instituciones de sus comunas en la construcción del futuro del distrito en aspectos del mejoramiento del desarrollo local y humano de la comuna.

La motivación hacia los vecinos de la comuna para su participación en la propuesta de proyectos de inversión que se incluyan en el presupuesto participativo, para el logro de un mejor y mayor desarrollo local y humano, es inexistente en los Gobiernos locales emergentes. Las autoridades una vez electas no se preocupan de este importante aspecto. La fuerza innovadora, creativa y de trabajo de la comunidad no es valorada como importante por las autoridades de turno. El problema subyace en la informalidad organizacional del vecindario local. En la medida que un Gobierno emergente no cuente con organizaciones sociales que reclamen y presionen sobre la orientación de los recursos ediles hacia la atención y solución de problemas con mayor prioridad para el consecuente beneficio de la comuna, los Gobiernos ediles de turno tendrán mayor margen de discrecionalidad para tomar decisiones muy alejadas de los intereses reales de la comuna.

No será posible que los Gobiernos locales emergentes logren desarrollar planes promocionales basados en liderazgo y motivación edil que estimule a la mayor participación vecinal en la formulación de propuestas de proyectos de inversión que se incluyan en el presupuesto participativo, si las municipalidades continúan aplicando estrategias deficientes de comunicación para la convocatoria de su comuna a participar en las sesiones y encuentros para la formulación y aprobación de presupuestos participativos.

No es posible ejercer liderazgo, ni mucho menos motivar a la comuna en los gobiernos locales emergentes, cuando las autoridades ediles no gozan de la confianza de la comuna. 


\section{REFERENCIAS BIBLIOGRÁFICAS}

"Alonso Gamero" (IUTAG), (2011) Santa Ana de Coro: Instituto Universitario de Tecnología

Agenda Municipal Latinoamericana (2011). Evento desarrollado del 28 a 29 de noviembre en la ciudad de San Francisco de Milagro- Ecuador. (2011)

Rosa Arlene María. (2009). ESTUDIO SOBRE BUENAS PRÁCTICAS DE LIDERAZGO POLÍTICO EN LA GESTIÓN MUNICIPAL EN REPÚBLICA DOMINICANA. Republica Dominicana.

Fabra, M.L. (1995) Técnicas de grupo para la cooperación. Barcelona. Ceac. Fundación Solidaridad. Brochure Informativo. Proyecto Participación e Incidencia de la Sociedad Civil en la Gestión Municipal.

GIRARDI, Giulio (2010). Desarrollo Local Sostenible, Poder Local Alternativo y Refundación de la Esperanza.
En: http://www.nodo50.org/venezuela-unida/ art_giulio_girardi01.htm\#girardi01.(2010) Pág.15

MANUAL DE LIDERAZGO PARA DIRECTIVOS. (2008). Del Centro De Estudios y Capacitación Cooperativa R.L.

Mario Rosales (1993). En su libro El Buen Gobierno Local y qué hacer para liderarlo. Pág. $N^{\circ} 51$.

Citado en "Los Secretos del Buen Alcalde", M. Rosales, IULA.( 2009) Quito, Página 39.

Rogers, C. (1996) El proceso de convertirse en persona. Barcelona. Editorial Paidós

Trechera, J.L. (2003) Trabajar en equipo: Talento y talante. España. Editorial Declee de Brouwner S.A. 